\title{
ON THE GEOMETRY OF GROSS-PITAEVSKI VORTEX CURVES FOR GENERIC DATA
}

\author{
JOSÉ ALBERTO MONTERO AND BENJAMIN K. STEPHENS
}

(Communicated by James E. Colliander)

\begin{abstract}
We study an energy functional that arises as a $\Gamma$-limit of the GrossPitaevskii (GP) energy. This last functional is often used to model rotating Bose-Einstein condensates, and the functional we study represents the contribution to the GP energy of vortices, or whirlpools, in the condensate. For our energy, we give a rough description of its (local) minimizers using ODE techniques along with an isoperimetric inequality.
\end{abstract}

\section{INTRODUCTION}

Bose-Einstein condensates (BEC) are a particular kind of matter sometimes characterized by the property that the particles making up a sample act as a single super-particle rather than as a collection of individual ones. An energy functional that is often used to model such a condensate is the Gross-Pitaevskii energy, which has been considered in several papers. (See for instance [6], [3, [5] and [7. See also [1] and 8] for good surveys on both mathematical and physical aspects of BEC.) An important feature of these condensates is that, when stirred, they develop whirlpools, often referred to as vortices. In dimension $n=3$, these vortices can be thought of as curves in space, and their contribution to the GP energy can be formally expressed as

$$
E_{0}(\gamma)=\int_{a}^{b}\left\{\rho(\gamma)\left|\gamma^{\prime}\right|+B_{0}(\gamma) \cdot \gamma^{\prime}\right\}
$$

Here $\Omega \subset \mathbb{R}^{3}$ represents the region occupied by the condensate, and $\left.\gamma:\right] a, b[\rightarrow \Omega$ is a Lipschitz curve with no boundary in $\Omega$ that models a vortex. $\rho$ is a real valued function representing a trapping potential that keeps the condensate in place, and $B_{0} \in C^{1,1}\left(\Omega ; \mathbb{R}^{3}\right)$ is a vector field determined both by the stirring applied to the condensate and the potential $\rho$.

From the references mentioned above, we know that the functional $E_{0}$ is in fact a $\Gamma$-limit of the GP energy. $\Gamma$-convergence is a convergence notion for functionals that yields various relations between minimizers of the functionals involved. For example, global minimizers of the converging functionals converge to global minimizers of the limiting one. Also, isolated local minimizers of the limit functional give raise to local minimizers of the converging functionals. In several works regarding the $\Gamma$-convergence of the GP energy to $E_{0}, \rho$ and $B_{0}$ were rotationally symmetric or explicit. However, the results in [7] show that this $\Gamma$-limit result is valid for rather

Received by the editors May 3, 2011 and, in revised form, January 16, 2012.

2010 Mathematics Subject Classification. Primary 49K15. 


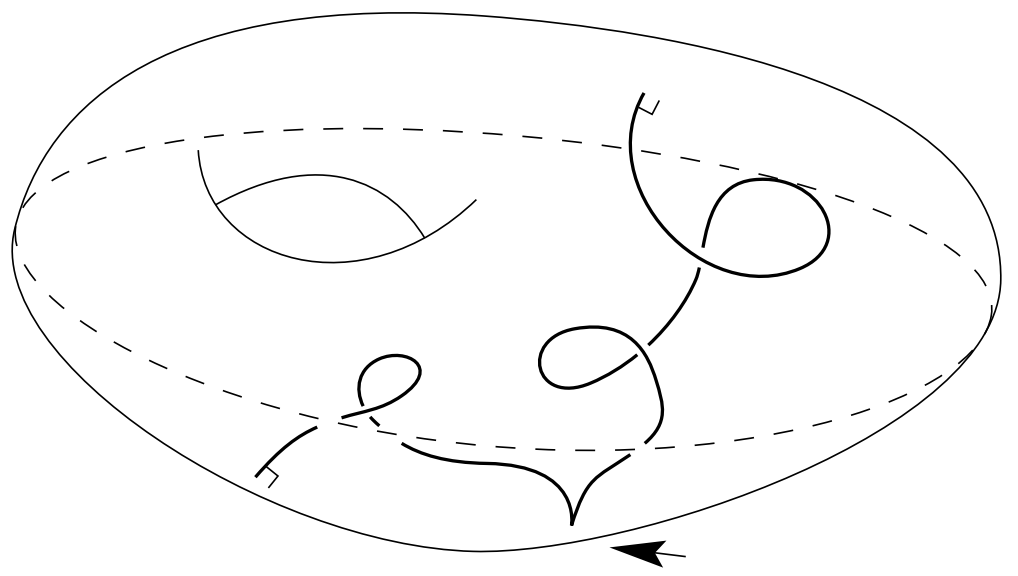

Figure 1. A critical point might have high curvature where its interior nears the boundary.

general $\rho$ and $B_{0}$. In all these cases $\rho$ and $D B_{0}$ vanish linearly at the boundary of $\Omega$, and we would like to study local minimizers of $E_{0}$ in this context.

1.1. Results. The first term in the energy $E_{0}$ is just the length of the curve $\gamma$ weighted by the function $\rho$, which we denote $L_{\rho}(\gamma)$. When $\rho$ vanishes at the boundary of $\Omega$, it is possible for curves to linger near the boundary, keeping bounded weighted length but attaining unbounded regular length. Our first result shows that this does not happen for critical points of $E_{0}$. Throughout this section we use the notation described in Section 2.1.

Theorem 1.1. Assume $\rho, D B_{0}$ vanishes to order $k$ at the boundary of $\Omega$ (made precise in hypotheses $(\mathrm{H} 1)-(\mathrm{H} 2))$. Let $J \in \mathcal{I}(\Omega)$ be a critical point of $E_{0}$ in the sense of (2.14) with $L_{\rho}(J)<\infty$, and let $\gamma$ be one of its regular irreducible Lipschitz curves. Then the curve $\gamma$ is $C^{1,1}$ in its interior and has bounded curvature. Furthermore, $\gamma$ has finite length, controlled by the weighted length

$$
L(\gamma)<A_{k} L_{\rho}(\gamma)
$$

via an explicit constant $A_{k}$ depending on the input data $\rho, B_{0}$, but independent of $\gamma$. The curve $\gamma$ is either a closed-loop in the interior of $\Omega$ or it meets the boundary of $\Omega$ at each of its ends, doing so perpendicularly.

Remark. We do not show any uniformity of the curvature bound on $\gamma$. Indeed, we expect that there exist critical points $J$ with high curvature where their interior nears the domain boundary (as indicated by the arrow in Figure 1).

With the regular length of critical points under control, it is possible to use the isoperimetric inequality for the domain $\Omega$ and (unweighted) length and area to rule out infinite-component local minimizers.

Theorem 1.2. Assume that $\partial \Omega$ is path-connected. Let $J$ be a local minimum of $E_{0}$ with respect to the flat metric, and assume that $J$ has finite weighted length. Then $J$ is a sum of finitely many $C^{1,1}$ curves and has finite total length according to (1.1). 
Under an additional assumption, we may use our tools to count the number of components.

Theorem 1.3. Assume that $\partial \Omega$ is path-connected. Let $J$ be a local minimum of energy $E_{0}$ with finite weighted length. If $J$ is moreover minimal in the sense that subtracting any component of $J$ increases energy, then $J$ has the following properties:

(i) There is a uniform lower bound on lengths:

$$
\|\rho\|_{L^{\infty}} L\left(\gamma_{i}\right) \geq L_{\rho}\left(\gamma_{i}\right) \geq L_{\min }:=\left(\|D B\|_{L^{\infty}} C_{i s o}(\Omega) A_{k}^{2}\right)^{-1}>0 .
$$

Here $C_{i s o}(\Omega)$ is an isoperimetric constant of the domain $\Omega$ and $A_{k}$ is given by Theorem 1.1 .

(ii) There is a bound on the number of components

$$
n_{\text {components }} \leq M_{\rho}(J) / L_{\text {min }}
$$

which is uniform in the sense that $L_{\text {min }}$ does not depend on $J$.

Remark 1.4. It is interesting to compare $E_{0}$ with the energy obtained from it by replacing $\rho$ by the function identically equal to 1 in $\Omega$. This energy also corresponds to a $\Gamma$-limit, but of the Ginzburg-Landau energy from super-conductivity instead. In this last case, critical points of $E_{0}$ obey an Euler-Lagrange equation which is the equation of motion of a charged particle moving in the magnetic field $\nabla \times B_{0}$, often referred to as motion under the Lorentz force.

Remark 1.5. Here we do not address the existence of curves that locally minimize $E_{0}$ for $\rho$ that vanishes on $\partial \Omega$. We know that such curves exist from concrete examples that can be found in [6] and [3], and our results apply to them.

\section{Setup}

The main data of our problem are the smooth domain $\Omega \subset \mathbb{R}^{3}$, the function $\rho$ and the vector field $B_{0}$. We assume that $\rho$ and $D B_{0}$ vanish to order $k$ at the boundary of $\Omega$, in the following sense:

(H1) $\rho=\omega^{k}$ for some integer $k \geq 1$ and a function $\omega \in C^{1,1}(\Omega ;] 0, \infty[)$ that satisfies $\omega=0$ on $\partial \Omega$ and in $\Omega$,

$$
\begin{aligned}
m<|\nabla \omega|^{2}+\omega & <M, \\
\left|D^{2} \omega\right| & <M_{2},
\end{aligned}
$$

for positive constants $m, M, M_{2}$.

(H2) $B_{0} \in C^{1,1}\left(\Omega ; \mathbb{R}^{3}\right)$ and

$$
\left|B_{0}\right|+\left|D B_{0}\right| \leq M_{3} \rho \text { in } \Omega,
$$

for some $M_{3}>0$.

Note in particular that the function $\omega \in C^{1,1}(\Omega ;] 0, \infty[)$ in (H1) has $\omega>0$ in $\Omega$, $\nabla \omega \neq 0$ on $\partial \Omega$, and satisfies

$$
C_{1} d_{\partial \Omega} \leq \omega \leq C_{2} d_{\partial \Omega}
$$

for $0<C_{1} \leq C_{2}$, where $d_{\partial \Omega}$ denotes the distance to the boundary function. The function $\omega$ could be taken to be a smoothed version of the distance to $\partial \Omega$. 
2.1. Line energy. To model the vortex curves we use rectifiable 1-currents. These are essentially countable sums of Lipschitz curves, in a way which we make precise in this section. Here we define the objects that we need in a somewhat non-standard way.

The space of 1-currents in $\Omega$ is defined as the dual of the space of smooth vector fields $C_{0}^{\infty}\left(\Omega ; \mathbb{R}^{3}\right)$ when this last space is endowed with the usual inductive limit topology. We denote such a space by $\mathcal{D}_{1}(\Omega)$. We say that $T \in \mathcal{D}_{1}(\Omega)$ is rectifiable if there are countably many (Lebesgue) measurable sets $I_{i} \subset \mathbb{R}$ and Lipschitz functions $\gamma_{i}: I_{i} \rightarrow \Omega$ such that

$$
T(B)=\sum_{i=1}^{\infty} \int_{I_{i}}\left\langle B \circ \gamma_{i}, \gamma_{i}^{\prime}\right\rangle
$$

for every $B \in C_{0}^{\infty}\left(\Omega ; \mathbb{R}^{3}\right)$. We use the notation $\langle\cdot, \cdot\rangle$ for the standard inner product in $\mathbb{R}^{3}$.

The boundary of $T$, denoted by $\partial T$, is the distribution acting on functions $\phi \in$ $C_{0}^{\infty}(\Omega)$ through the formula

$$
(\partial T)(\phi)=T(\nabla \phi) .
$$

A norm on $\mathcal{D}_{1}(\Omega)$ that we use frequently in this paper is the mass. For rectifiable 1-currents, which are essentially countable families of Lipschitz curves, this mass is just the total length of the curves. We require a weighted version of this that we denote by $M_{\rho}(T)$ and that can be expressed as

$$
M_{\rho}(T)=\sup \left\{T(B): B \in C_{0}^{\infty}\left(\Omega ; \mathbb{R}^{3}\right),|B| \leq \rho \text { in } \Omega\right\} .
$$

We will always work with rectifiable 1-currents with zero boundary and finite weighted mass, and we will denote the collection of such currents by $\mathcal{I}(\Omega)$. In other words,

$$
\mathcal{I}(\Omega)=\left\{T \in \mathcal{D}_{1}(\Omega): T \text { is rectifiable, } M_{\rho}(T)<\infty, \partial T=0\right\} .
$$

The following version of 4.2.25 from [4] can be found in [6].

Lemma 2.1. Let $T \in \mathcal{I}(\Omega)$. One can choose the $I_{i}$ and the curves $\gamma_{i}$ in (2.5) in such a way that $\left.I_{i}=\right] a_{i}, b_{i}[$ is an interval, possibly infinite,

$$
M_{\rho}(T)=\sum_{i=1}^{\infty} \int_{a_{i}}^{b_{i}} \rho\left(\gamma_{i}\right)\left|\gamma_{i}^{\prime}\right|,
$$

and each curve $\gamma_{i}: I_{i} \rightarrow \Omega$ is injective and of one of the following two types:

(i) Closed loop. The following limits exist, belong to the interior of $\Omega$ and agree:

$$
\lim _{t \rightarrow a_{i}^{+}} \gamma_{i}(t)=\lim _{t \rightarrow b_{i}^{-}} \gamma_{i}(t)
$$

(ii) Boundary-to-boundary curve:

$$
\lim _{t \rightarrow a_{i}^{+}} \omega\left(\gamma_{i}(t)\right)=\lim _{t \rightarrow b_{i}^{-}} \omega\left(\gamma_{i}(t)\right)=0 .
$$

Definition 2.2. We call each $\gamma_{i}$ in the lemma above an irreducible component of $T$.

Next, we wish to endow $\mathcal{I}(\Omega)$ with a metric, usually referred to as the flat metric. To do this we need first to recall a few definitions. 
Definition 2.3. A set $S \subset \mathbb{R}^{3}$ is called 2-rectifiable if there is a countable collection of bounded sets $A_{k} \subset \mathbb{R}^{2}$, along with Lipschitz functions $f_{k}: A_{k} \rightarrow \mathbb{R}^{3}, k \geq 1$, such that

$$
H^{(2)}\left(S \backslash \bigcup_{k=1}^{\infty} f_{k}\left(A_{k}\right)\right)=0 .
$$

Here, and throughout the paper, $H^{(2)}$ denotes the 2 dimensional Hausdorff measure.

Remark 2.4. It is well known that when $S \subset \mathbb{R}^{3}$ is 2-rectifiable and $H^{(2)}$-measurable, at $H^{(2)}$-almost every $x \in S, S$ possesses an approximate tangent plane. Because we are in $\mathbb{R}^{3}$, this is equivalent to saying that at almost every $x \in S$ there is a vector $\nu_{x}$ normal to $S$ which is $H^{(2)}$-measurable (cf. 3.2 .19 and 3.2.25 of [4]).

Definition 2.5. Given a 1-current $T \in \mathcal{I}(\Omega)$ and a 2-rectifiable, $H^{(2)}$-measurable set $S \subset \Omega$, we say that $T=\partial S$ (relative to $\Omega$ ) if, for every $B \in C_{0}^{\infty}\left(\Omega ; \mathbb{R}^{3}\right)$, we have the identity

$$
T(B)=\int_{S}\langle\nu, \nabla \times B\rangle d H^{(2)} .
$$

Here $\nu$ denotes the normal vector to $S$, and $\nabla \times B$ the curl of $B$.

With these definitions in hand, we can now define the flat metric: for $T_{1}, T_{2} \in$ $\mathcal{I}(\Omega)$, define

$$
\begin{array}{r}
d_{b}\left(T_{1}, T_{2}\right)=\inf \left\{H^{(2)}(\Sigma): \Sigma \subset \Omega, 2 \text {-rectifiable, } H^{(2)}\right. \text {-measurable } \\
\text { such that } \left.\partial \Sigma=T_{1}-T_{2}\right\} .
\end{array}
$$

We define $d_{b}\left(T_{1}, T_{2}\right)=\infty$ if the set on the right-hand side of (2.11) is empty. We study the local minima of

$$
E_{0}(T)=M_{\rho}(T)+T\left(B_{0}\right)
$$

in the sense of $d_{b}$, where $B_{0}$ is the vector field from (H2). Note that when a current $T \in \mathcal{I}(\Omega)$ is comprised by a single Lipschitz curve $\gamma:] a, b[\rightarrow \Omega$, this definition becomes

$$
E_{0}(\gamma)=L_{\rho}(\gamma)+\int_{a}^{b}\left\langle B(\gamma), \gamma^{\prime}\right\rangle d t
$$

where $L_{\rho}$ denotes weighted length

$$
L_{\rho}(\gamma)=\int_{a}^{b} \rho(\gamma)\left|\gamma^{\prime}\right| .
$$

This is the form of $E_{0}$ that we used in the discussion of the introduction. Note that the terms in the sum of the right-hand side of (2.9) can be interpreted as the weighted lengths of the irreducible components of $T$, mentioned in Definition 2.2 .

Recall now that the first variation of $E_{0}$ at the curve $\gamma$ in the direction of a smooth vector field $Y \in C_{0}^{\infty}(] a, b\left[, \mathbb{R}^{3}\right)$, which we denote by $Y_{*} E_{0}(\gamma)$, is defined by the formula

$$
Y_{*} E_{0}(\gamma)=\left.\frac{d}{d \xi}\right|_{\xi=0} E_{0}(\gamma+\xi Y) .
$$

Note that for sufficiently small $\xi, \gamma+\xi Y$ maps the (compact) support of $Y$ to a compact subset of $\Omega$ that lies at a positive distance from $\partial \Omega$. Thus for $\xi$ small enough, $t \mapsto \gamma(t)+\xi Y(t)$ is a curve lying in $\Omega$ and is in the domain of $E_{0}$. 
Definition 2.6. We say $T \in \mathcal{I}(\Omega)$ is a critical point of $E_{0}$ with respect to interior variations if each irreducible component $\gamma:] a, b[\rightarrow \Omega$ of $T$ has

$$
Y_{*} E_{0}(\gamma)=0
$$

for all $Y \in C_{0}^{\infty}(] a, b\left[, \mathbb{R}^{3}\right)$.

Remark 2.7. We remark that if $T \in \mathcal{I}(\Omega)$ is a local minimum of $E_{0}$ with respect to $d_{b}$, then it must be a critical point in the above sense. This is because as $\xi \rightarrow 0$, and for every $\gamma$ irreducible component of $T$, the curves $\gamma_{\xi}=\gamma+\xi Y$ may be connected to $\gamma$ by ruled surfaces of arbitarily small area.

\section{Euler-Lagrange EQUATION AND FINITENESS OF LENGTH}

In this section we show how weighted length controls length for critical curves of $E_{0}$, proving (1.1) of Theorem 1.1. The key idea is to vary large subarcs of such a curve in the direction of $\nabla \rho$.

Consider an irreducible component $\Gamma$ of $J_{0}$, a Lipschitz map with $\Gamma: I \rightarrow \mathbb{R}^{3}$, where $I$ is a possibly unbounded interval, and $L_{\rho}(\Gamma)$ finite. Pick $t_{0} \in I$ and consider the signed arc-length of $\gamma$,

$$
s(t)=\int_{t_{0}}^{t}\left|\Gamma^{\prime}\right|(\bar{t}) d \bar{t}
$$

Let $\gamma: I^{\prime} \rightarrow \mathbb{R}^{3}$ be the reparameterized curve, $\gamma(s(t))=\Gamma(t)$. Set $s_{a}=\lim _{t \rightarrow a^{+}} s(t)$ and $s_{b}=\lim _{t \rightarrow b^{-}} s(t)$ so that $\left.I^{\prime}=\right] s_{a}, s_{b}\left[\right.$. Note that $I^{\prime}$ may be unbounded.

As in Section 2, we consider a vector field $V \in C_{0}^{\infty}\left(I^{\prime} ; \mathbb{R}^{3}\right)$ and a family of curves

$$
\gamma_{\xi}(s)=\gamma(s)+\xi V(s) .
$$

A standard computation shows that

$$
V_{*} E_{0}(\gamma)=\int_{s_{a}}^{s_{b}}\left\{\langle V, \nabla \rho\rangle+\rho\left\langle\frac{d V}{d s}, \frac{d \gamma}{d s}\right\rangle+\left\langle\left(D B_{0}\right) V, \frac{d \gamma}{d s}\right\rangle+\left\langle B_{0}, \frac{d V}{d s}\right\rangle\right\} .
$$

Integrating the last term by parts we obtain

$$
V_{*} E_{0}(\gamma)=\int_{s_{a}}^{s_{b}}\left\{\rho\left\langle\frac{d V}{d s}, \frac{d \gamma}{d s}\right\rangle+\left\langle V, \nabla \rho+\left(D B_{0}^{T}-D B_{0}\right) \frac{d \gamma}{d s}\right\rangle\right\} .
$$

Thus the condition $V_{*} E_{0}(\gamma)=0$ becomes the weak ODE

$$
\frac{d}{d s}\left\{\rho(\gamma) \frac{d \gamma}{d s}\right\}=\nabla \rho+\left(D B_{0}^{T}-D B_{0}\right) \frac{d \gamma}{d s}
$$

for $\gamma$. By standard regularity for ODEs (see for instance chapter VIII of [2]) we obtain that $\gamma \in C_{l o c}^{1,1}(] s_{a}, s_{b}[)$. It is a simple matter to check that the last equation for $\gamma$ in dimension 3 can be re-written as

$$
\frac{d}{d s}\left\{\rho(\gamma) \frac{d \gamma}{d s}\right\}=\nabla \rho+\frac{d \gamma}{d s} \times \nabla \times B_{0} .
$$

We are now in a position to prove (1.1). To do this, we will establish that for every integer $j \in\{0, \ldots, k-1\}$, we have

$$
\int_{s_{a}}^{s_{b}} \omega^{k-j-1} \leq A_{j} \int_{s_{a}}^{s_{b}} \omega^{k-j},
$$

for some constant $A_{j}$ that depends only on $\rho$ and $B_{0}$. Note that this reduces the power in $\rho$ successively from $k$ all the way down to 0 . 
We establish (3.5) by varying a piece of $\gamma$ in direction $\nabla \omega$. In ODE terms this means taking the inner product of (3.3) with the vector field $z(s)=\nabla \omega(\gamma(s))$ and then integrating on $\left[s_{0}, s_{1}\right] \subset I^{\prime}$ :

$$
-\int_{s_{0}}^{s_{1}} \rho(\gamma)\left\langle\frac{d \gamma}{d s}, \frac{d z}{d s}\right\rangle=\int_{s_{0}}^{s_{1}}\left\langle z, \nabla \rho+\frac{d \gamma}{d s} \times \nabla \times B_{0}\right\rangle-\left.\rho(\gamma)\left\langle\frac{d \gamma}{d s}, z\right\rangle\right|_{s_{0}} ^{s_{1}} .
$$

Recalling hypothesis (H1), we obtain from (3.6) that

$$
\begin{aligned}
k \int_{s_{0}}^{s_{1}} \omega^{k-1}|\nabla \omega|^{2}(\gamma) & =-\int_{s_{0}}^{s_{1}}\left\{\rho(\gamma)\left\langle\frac{d \gamma}{d s}, D^{2} \omega \frac{d \gamma}{d s}\right\rangle+\left\langle(\nabla \omega)(\gamma), \frac{d \gamma}{d s} \times \nabla \times B_{0}\right\rangle\right\} \\
& +\left.\rho(\gamma)\left\langle\frac{d \gamma}{d s},(\nabla \omega)(\gamma)\right\rangle\right|_{s_{0}} ^{s_{1}} .
\end{aligned}
$$

Now by Lemma 2.1, $\gamma$ falls into one of two cases:

(i) It is a closed loop. In this case, its image lies a positive distance away from $\partial \Omega$. So $\rho(\gamma(t))>c>0$ and $L(\gamma)<c^{-1} L_{\rho}(\gamma)$. We may reparametrize $\gamma$ by arclength to get a curve $\gamma: I^{\prime} \rightarrow \mathbb{R}^{3}$ on the finite interval $I^{\prime}=$ $\left[s_{a}, s_{b}\right]$. Moreover, we must have $\gamma^{\prime}$ agreeing at the endpoint of $I^{\prime}$. (To see this we could reparametrize $\gamma$ under a cyclic shift and use the above ODE regularity again.)

(ii) It is "boundary to boundary", and we get

$$
\lim _{s \rightarrow s_{a}} \omega(\gamma(s))=\lim _{s \rightarrow s_{b}} \omega(\gamma(s))=0
$$

Then, taking the limits as $s_{0} \rightarrow s_{a}^{+}$and $s_{1} \rightarrow s_{b}^{-}$, the boundary terms in (3.7) vanish and we obtain

$$
k \int_{s_{a}}^{s_{b}} \omega^{k-1}|\nabla \omega|^{2}(\gamma) \leq\left\{\left\|D^{2} \omega\right\|_{\infty}+\|\nabla \omega\|_{\infty}\left\|\left(\nabla \times B_{0}\right) / \rho\right\|_{\infty}\right\} \int_{s_{a}}^{s_{b}} \rho(\gamma) .
$$

We now recall hypothesis (H1) (equation (2.1) ) and find

$$
\int_{s_{a}}^{s_{b}}\left\{\omega^{k-1}|\nabla \omega|^{2}(\gamma)+\omega^{k}\right\} \geq m \int_{s_{a}}^{s_{b}} \omega^{k-1}
$$

These last two estimates imply that

$$
\begin{aligned}
\int_{s_{a}}^{s_{b}} \omega^{k-1} & \leq \frac{1}{m k}\left\{k+\left\|D^{2} \omega\right\|_{\infty}+\|\nabla \omega\|_{\infty}\left\|\left(\nabla \times B_{0}\right) / \rho\right\|_{\infty}\right\} \int_{s_{a}}^{s_{b}} \rho(\gamma) \\
& =A_{1} L_{\rho}(\gamma)
\end{aligned}
$$

where $A_{1}$ is a constant that depends on $k, \rho$ and $B_{0}$. We have established (3.5) for $j=0$.

We now iterate this procedure as follows: choose $0 \leq j \leq k-1$ and set

$$
z(s)=\omega^{-j}(\gamma)(\nabla \omega)(\gamma(s))
$$


in (3.6). After some re-arrangement, one obtains

$$
\begin{aligned}
\int_{s_{0}}^{s_{1}} \omega^{k-1-j}\left\{k|\nabla \omega|^{2}(\gamma)-j\left(\nabla \omega \cdot \frac{d \gamma}{d s}\right)^{2}\right\} & =\int_{s_{0}}^{s_{1}} \omega^{-j}(\gamma)\left\langle\frac{d \gamma}{d s}, \nabla \omega \times \nabla \times B_{0}\right\rangle \\
& -\int_{s_{0}}^{s_{1}} \omega^{k-j}\left\langle D^{2} \omega \frac{d \gamma}{d s}, \frac{d \gamma}{d s}\right\rangle \\
& -\left.\omega^{k-j}(\gamma)\left\langle\frac{d \gamma}{d s}, D^{2} \omega \frac{d \gamma}{d s}\right\rangle\right|_{s_{0}} ^{s_{1}} .
\end{aligned}
$$

Here we now recall that $j \in\{0, \ldots, k-1\}$, that $\left|\frac{d \gamma}{d s}\right|=1$, that $\left|D^{2} \omega\right|$ is bounded, and that (2.3) implies $\left|\nabla \times B_{0}\right| \leq M_{3} \rho=M_{3} \omega^{k}$. We let $s_{0} \rightarrow s_{a}^{+}$and $s_{1} \rightarrow s_{b}^{-}$to obtain (3.5). A straightforward induction argument now shows that for $\rho=\omega^{k}$

$$
L(\gamma) \leq A_{k} L_{\rho}(\gamma)
$$

where again $A_{k}$ depends on $\rho, B_{0}$ and $k$.

\section{Behavior NeAR BOUNDARY}

In this section we want to determine the behavior near $\partial \Omega$ of a boundary-toboundary critical curve $\gamma$. In order to do this, we note that, by the previous section, $\gamma$ has finite length, and in the notation of the previous section, both the limits

$$
\lim _{s \rightarrow s_{a}^{+}} \gamma(s) \text { and } \lim _{s \rightarrow s_{b}^{-}} \gamma(s)
$$

exist and in fact belong to $\partial \Omega$. We show next that whenever this happens, $\gamma$ meets $\partial \Omega$ perpendicularly at these points. Note that we can choose $s_{a}=0$ and $\left.s_{b} \in\right] 0, \infty[$, which we do from now on. Choose $s \in] 0, s_{b}[$, and integrate the equation (3.4) over ] $0, s\left[\right.$. Because $\omega=0$ on $\partial \Omega$ and $\left|\gamma^{\prime}\right| \equiv 1$, we obtain

$$
\omega^{k} \gamma^{\prime}=k \int_{0}^{s} \omega^{k-1} \nabla \omega d \xi+\int_{0}^{s} T_{B} \gamma^{\prime} d \xi
$$

Here we use the notation $T_{B}=D B^{T}-D B$, and abbreviate $\omega(\gamma(s)),(\nabla \omega)(\gamma(s))$, etc., by $\omega(s)$, and so on. This implies that

$$
\begin{aligned}
\omega^{k} \gamma^{\prime} & =k \int_{0}^{s} \omega^{k-1}(\xi) d \xi(\nabla \omega)(s) \\
& +k \int_{0}^{s} \omega^{k-1}(\xi)((\nabla \omega)(\xi)-(\nabla \omega)(s)) d \xi+\int_{0}^{s} T_{B} \gamma^{\prime} d \xi
\end{aligned}
$$

Next, note that

$$
|(\nabla \omega)(\xi)-(\nabla \omega)(s)| \leq \sup _{x \in \Omega}\left|\left(D^{2} \omega\right)(x)\right| s
$$

and

$$
\left|T_{B}(\xi)\right| \leq C \omega^{k}(\xi) \leq C s \omega^{k-1}(\xi)
$$

both for all $\xi \in] 0, s[$. Denoting the orthogonal projection onto the vector space orthogonal to $\gamma^{\prime}$ by $\pi_{\gamma^{\prime} \perp}$, we obtain from (4.1) that

$$
\begin{aligned}
\pi_{\gamma^{\prime} \perp} \nabla \omega(s) & =\frac{-1}{\int_{0}^{s} \omega^{k-1} d \xi} \pi_{\gamma^{\prime \perp}} \int_{0}^{s} \omega^{k-1}(\xi)((\nabla \omega)(\xi)-(\nabla \omega)(s)) d \xi \\
& +\frac{-1}{k \int_{0}^{s} \omega^{k-1} d \xi} \pi_{\gamma^{\prime \perp}} \int_{0}^{s} T_{B} \gamma^{\prime} .
\end{aligned}
$$


From this last identity we obtain

$$
\left|\pi_{\gamma^{\prime} \perp}(\nabla \omega)(s)\right| \leq C s,
$$

so that as $s \rightarrow 0, \pi_{\gamma^{\prime \perp}}(\nabla \omega)(s) \rightarrow 0$. This shows that $\gamma^{\prime}$ and $\nabla \omega$ become parallel as $s \rightarrow 0$ (recall that $\nabla \omega \neq 0$ near $\partial \Omega$ ). Since $\omega=0$ on $\partial \Omega$ and $\omega$ is smooth, $\nabla \omega$ on $\partial \Omega$ is parallel to the normal of this last set. This shows that whenever $\gamma$ meets $\partial \Omega$, it does so at a $90^{\circ}$ angle.

4.1. Curvature. We show next that the curvature of $\gamma$ is bounded. To do this we note that, by (H1) and (2.4), we can always find $\mu>0$ so that $|\nabla \omega| \geq \mu$ in the set

$$
\Omega_{\mu}=\{x \in \Omega: \operatorname{dist}(x ; \partial \Omega) \leq \mu\} .
$$

Then, for $s^{*}>0$ such that $0<\max \{C, 1\} s^{*} \leq \mu / 2$, where $C>0$ is the constant from (4.3) and all $s \in] 0, s^{*}[$, we have

$$
\begin{aligned}
\left|\left(I-\pi_{\gamma^{\prime}}\right) \nabla \omega\right|(s) & =\left(|\nabla \omega|^{2}-\left|\pi_{\gamma^{\prime}}(\nabla \omega)(s)\right|^{2}\right)^{1 / 2} \\
& \geq\left(\mu^{2}-\mu^{2} / 4\right)^{1 / 2} \geq \frac{\sqrt{3}}{2} \mu .
\end{aligned}
$$

In particular, $(\nabla \omega)(s) \cdot \gamma^{\prime}(s) \geq \sqrt{3} \mu / 2$ for all $\left.s \in\right] 0, s^{*}[$, and then

$$
\omega(s)=\int_{0}^{s}(\nabla \omega)(\xi) \cdot \gamma^{\prime}(\xi) d \xi \geq \frac{\sqrt{3} \mu}{2} s .
$$

We note next that (3.4) can be re-written as

$$
\gamma^{\prime \prime}=\frac{k}{\omega} \pi_{\gamma^{\prime \perp}} \nabla \omega+\omega^{-k} T_{B} \gamma^{\prime} .
$$

Recalling that $T_{B}=D B^{T}-D B$, we apply (2.3), (4.3) and (4.5) in this last identity to obtain

$$
\left|\gamma^{\prime \prime}\right| \leq C
$$

for all $s \in] 0, s^{*}$. The same argument applies near $s_{1}>0$. Finally, we may assume that away from the endpoints of $] 0, s_{1}[, \gamma$ remains away from $\partial \Omega$, for otherwise we just shrink the interval $] 0, s_{1}$ [ enough to satisfy this property. This shows that $\left|\gamma^{\prime \prime}\right|$ is uniformly bounded in $] 0, s_{1}$ [, which implies that $\gamma$ has bounded curvature. This concludes the proof of the theorem.

\section{Finiteness}

In this section we prove Theorems 1.2 and 1.3 . Throughout this section we assume that $\partial \Omega$ is path-connected.

Lemma 5.1. If $\gamma$ is a critical point of $E_{0}$ in the sense of Definition 2.6, then either

$$
L_{\rho}(\gamma) \geq L_{\min }:=\left(\|D B\|_{L^{\infty}} C_{i s o}(\Omega) A_{k}^{2}\right)^{-1}
$$

or $E_{0}(\gamma)>0$.

Proof. Let $\gamma \in \mathcal{I}(\Omega)$ be a critical point of $E_{0}$. The assumption that $\partial \Omega$ is pathconnected allows us to appeal to the Isoperimetric Inequality (for instance 4.5.14 of [4] will do) to obtain a 2 -rectifiable set $\Sigma$ with $\partial \Sigma=\gamma$ relative to $\Omega$ and with

$$
H^{(2)}(\Sigma) \leq C_{\text {iso }}(\Omega) L(\gamma)^{2} .
$$


We may use Stokes' theorem in the second term of $E_{0}$ to estimate the energy as follows:

$$
E_{0}(\gamma) \geq L_{\rho}(\gamma)-\|D B\|_{L^{\infty}} C_{i s o}(\Omega) L(\gamma)^{2}
$$

But then we apply Theorem 1.1 to find

$$
E_{0}(\gamma) \geq L_{\rho}(\gamma)-\|D B\|_{L^{\infty}} C_{i s o}(\Omega) A_{k}^{2} L_{\rho}(\gamma)^{2}
$$

So we see that if

$$
L_{\rho}(\gamma)<L_{\min }:=\left(\|D B\|_{L^{\infty}} C_{i s o}(\Omega) A_{k}^{2}\right)^{-1},
$$

then (5.1) reveals that $\gamma$ contributes positive energy.

We now prove our last two theorems.

Proof of Theorem 1.2. Let $\epsilon>0$. Because $L_{\rho}(J)$ is finite we know that if $J$ has infinitely many irreducible components, we may find one $\gamma^{*}$ with weighted length less than $\epsilon$. Because $J$ is a local minimum with respect to the flat metric, we know $\gamma^{*}$ is a critical point in the sense of Definition 2.6 (see the remark after the definition). By the Isoperimetric Inequality, there is a 2-rectifiable set $\Sigma$ with $\partial \Sigma=\gamma$ and

$$
H^{(2)}(\Sigma)<C_{\text {iso }}(\Omega) L(\gamma)^{2}<C_{\text {iso }}(\Omega) A_{k} \epsilon^{2} .
$$

By Lemma 5.1, for small enough $\epsilon$, we have $E_{0}\left(\gamma^{*}\right)>0$, so $J-\gamma$ has less energy. Also, by definition, $d_{b}(J-\gamma, J) \leq H^{(2)}(\Sigma)$. So taking $\epsilon$ to zero we find rectifiable 1 -currents which are arbitrarily close to $J$ in the flat metric and have strictly less energy. This contradiction proves the theorem.

Proof of Theorem 1.3. Claim (i) follows from Lemma 5.1. Claim (ii) follows from the fact that every component has non-negative weighted length, and if there were more than the claimed number, this would violate Lemma 5.1.

\section{ACKNOWLEDGMENTS}

The first author wishes to thank his sponsor, R. L. Jerrard, for suggesting this problem and for many helpful conversations on the subject.

The second author would like to thank his sponsors, A. Burchard, R. L. Jerrard, R. J. McCann, I. M. Sigal and C. Sulem (via NSERC Discovery grants RGPIN 2117006-03, 311685-05, 7901-07, 46179-05, and 261955-03). He also thanks the Young Mathematicians Conference, Centre de Recherches Mathématiques, Montreal, January 2008.

\section{REFERENCES}

1. Aftalion, A., Vortices in Bose-Einstein Condensates, Progress in Nonlinear Differential Equations and Their Applications, Birkhäuser, 2006. MR2228356 (2007g:82078)

2. Brezis, H., Analyse fonctionnelle, deuxième edition, Dunod, 1999.

3. Alama, S., Bronsard, L. and Montero, A., Vortices for a rotating toroidal Bose-Einstein condensate, Arch. Ration. Mech. Anal. 187 (2008), no. 3, 481-522. MR2372812 (2009d:82015)

4. Federer, H., Geometric measure theory, Springer-Verlag, 1969. MR0257325 (41:1976)

5. Ignat, R. and Millot, V., Energy expansion and vortex location for a two-dimensional rotating Bose-Einstein condensate, Rev. Math. Phys. 18 (2006), 119-162. MR.2228922 (2007b:58030)

6. Jerrard, R. L., Local minimizers with vortex filaments for a Gross-Pitaevsky functional, ESAIM Control Optim. Calc. Var. 13 (2007), no. 1, 35-71 (electronic). MR.2282101 (2008g:58026) 
7. Montero, J. A., Hodge decomposition with degenerate weights and the Gross-Pitaevskii energy. J. Funct. Anal. 254 (2008), no. 7, 1926-1973. MR.2397879 (2009h:35028)

8. Pitaevskii, L. and Stringari, S., Bose-Einstein condensation, Oxford University Press, 2003. MR2012737 (2004i:82038)

Facultad de Matemáticas, Pontificia Universidad Católica de Chile, Vicuña MacKenna 4860, Santiago, Chile

E-mail address: amontero@mat.puc.cl

Department of Mathematics, University of Washington, Seattle, Washington 98195 E-mail address: bensteph@math.washington.edu

$U R L$ : www. bkstephens.net 